\title{
Consequences of Ventromedial Hypothalamic Lesions upon Insulin and Glucagon Secretion by Subsequently Isolated Perfused Pancreases in the Rat
}

\author{
Françoise Rohner-Jeanrenaud and Bernard Jeanrenaud, Laboratoires de \\ Recherches Médicales and the Department of Medicine, Geneva University \\ Medical School, Geneva, Switzerland
}

A B S TRACT The existence of a relationship between the ventromedial hypothalamic area $(\mathrm{VMH})$ and the activity of the endocrine pancreas has been shown previously. This relationship has been further tested and extended in the present study, using isolated perfused pancreases from rats previously lesioned (4-7 d) in the VMH. It was found that in isolated pancreases obtained from rats fed ad lib. for $4 \mathrm{~d}$ after VMH lesions (i.e., that were hyperphagic), the typical biphasic pattern of insulin secretion was observed following glucose stimulation $(20 \mathrm{mM})$ and that the total insulin output was much greater than that of controls. The increased insulin output was not a result of hyperphagia because similar results were obtained using pancreases obtained from VMH-lesioned rats in which a food restriction matching exactly that of control rats was started either immediately or $3 \mathrm{~d}$ after the lesions. Pancreases from such food-restricted VMHlesioned rats oversecreted insulin, when compared with controls fed the same amount, from $7 \mathrm{mM}$ of glucose concentration in perfusion medium onwards. After the addition of arginine $(10 \mathrm{mM})$, the total output of glucagon by pancreases from food-restricted $\mathrm{VMH}-$ lesioned rats was twice that of controls. Qualitatively, the arginine-induced glucagon secretion by pancreases from food-restricted VMH-lesioned rats retained its biphasic pattern. Similarly, epinephrine $(0.1 \mu \mathrm{M})$ elicited a greater glucagon release by pancreases from food-restricted VMH-lesioned rats when compared with controls. These data further support the concept of a link (as yet undefined) between the hypothalamus and the endocrine pancreas, as lesions of the VMH area resulted in abnormal secretion not only of insulin, but of glucagon as well.

Received for publication 24 July and in revised form 30 November 1979.

\section{INTRODUCTION}

Chemical or electrolytic lesions of the ventromedial hypothalamus $(\mathrm{VMH})^{1}$ have been shown to produce obesity in several species (1-4). Although the VMH syndrome is often accompanied by hyperphagia (5), lesions effective in causing obesity in the absence of overeating have suggested that factors other than hyperphagia also contributed to the occurrence of this type of obesity $(6,7)$.

Insufficient growth hormone production $(6,8,9)$, as well as hypoactivity and pituitary insufficiency leading to hypothyroidism have been demonstrated in $\mathrm{VMH}-$ lesioned animals $(10,11)$. However, among the observed endocrine changes, hyperinsulinemia has been found to be the most prominent one $(12,13)$. Thus, VMH lesions failed to induce weight gain in diabetic animals $(14,15)$, suggesting the absolute requirement of intact $B$ cells in the establishment and maintenance of the obesity syndrome (15). The observation that hypothalamic obesity could not be produced in rats in which B cells had been destroyed by streptozotocin administration and replaced by fetal pancreas transplants devoid of intact innervation (16), supported the concept of a direct neural influence on the B-cell function. Analogous conclusions were drawn on the basis of chronic experiments carried out in VMHlesioned rats with superimposed vagotomy (17-19). More recently it was shown that bilateral subdiaphragmatic vagotomy performed 50 min after VMH lesions, immediately and completely reversed the observed glucose-induced hyperinsulinemia (20). It is well established that the stimulation of the vagus nerve increases both insulin and glucagon output by the pan-

\footnotetext{
${ }^{1}$ Abbreviations used in this paper: IRG, immunoreactive glucagon; IRI, immunoreactive insulin; $\mathrm{VMH}$, ventromedial hypothalamus.
} 
creas (21-23). Thus, based on the hypothesis of a vagally-mediated hyperinsulinemia in VMH-lesioned animals, one would anticipate changes not only in insulin secretion but in glucagon secretion as well. A combined oversecretion of insulin and glucagon in VMH-lesioned animals would help to explain the recent observations that perfused livers from these animals have increased deamination of amino acids, once taken up, and a diversion of the deaminated intermediates toward lipid synthesis pathways concomitantly with an increase in urea production (24-26).

The present studies were undertaken to further substantiate the concept of the relationship between the hypothalamus and the secretory activity of the endocrine pancreas, using isolated perfused pancreases from normal and previously VMH-lesioned rats, and measuring not only insulin secretion but that of glucagon as well.

\section{METHODS}

Animals. 8-10 wk-old female Wistar rats, weighing between 200 and $240 \mathrm{~g}$ and bred in our laboratories, were used. They were placed in animal quarters with a constant temperature $\left(23^{\circ} \mathrm{C}\right)$ and a fixed 12 -h light cycle. They were fed with standard laboratory chow (UAR Laboratories, Villemoisson, Epinay/Orge, France).

Electrolytic lesion of the hypothalamus. Bilateral stereotaxic lesions of the VMH (stereotaxic apparatus, David Kopf Instruments, Tujunga, Calif., model 900) were made electrolytically in rats anesthetized with ketamine hydrochloride $(80 \mathrm{mg} / \mathrm{kg}$ body wt). The stereotaxic coordinates used were selected according to De Groot (27): anterior-posterior $(\mathrm{AP})+6.0$, lateral $\pm 0.6-0.8$, and ventral -2.8 . Anodal DC ( $1 \mathrm{~mA}$ for $30 \mathrm{~s}$ ) was passed through epoxy-coated stainless steel electrodes $(0.4 \mathrm{~mm}$ Diam with bared tip of $0.2 \mathrm{~mm}$ ). Unoperated rats were used as controls for the following reasons: (a) no difference in glucose-induced insulin secretion was found between perfused pancreases from unoperated or sham-operated rats (i.e., rats treated as the lesioned animals except that no current was passed through the electrodes); (b) it was observed that sham operation actually decreased food intake on the day after the operation (28).

Feeding conditions of rats. Perfusion of pancreases from control and VMH-lesioned rats was carried out using three different feeding conditions.

A. In the first, pancreases were isolated $4 \mathrm{~d}$ after VMHlesions from 9-wk-old rats fed ad lib. As these rats became hyperphagic after VMH-lesioning and were heavier than controls (mean increase over controls: $30 \pm 6 \mathrm{~g}, n=5$ ), they were compared with 10 wk-old controls, so that the body weights of both groups were almost identical (controls: $226 \pm 5 \mathrm{~g}, n=14$; V.MH-lesioned rats: $235 \pm 7 \mathrm{~g}, n=5$; NS).

B. In the second experimental condition, $8 \mathrm{wk}$-old rats were used. As shown by Fig. 1A, VMH-lesioned rats were first fed ad lib. for $3 \mathrm{~d}$ to detect successful lesions of the VMH area by the occurrence of hyperphagia and increased body weight (mean excess in body weight over controls after $3 \mathrm{~d}: 44 \pm 5 \mathrm{~g}, n=7$ ). Subsequently (Fig. 1A), control and VMH-lesioned rats were fed for $4 \mathrm{~d}$ the same amount of food using an automatic food distributor that delivered weightstandardized pellets $(3 \mathrm{~g})$ every $6 \mathrm{~h}$. Total food intake (i.e., $12 \mathrm{~g} / \mathrm{d}$ ) was calculated to be less, by $25 \%$, than the usual

\section{A}

CONTROLS
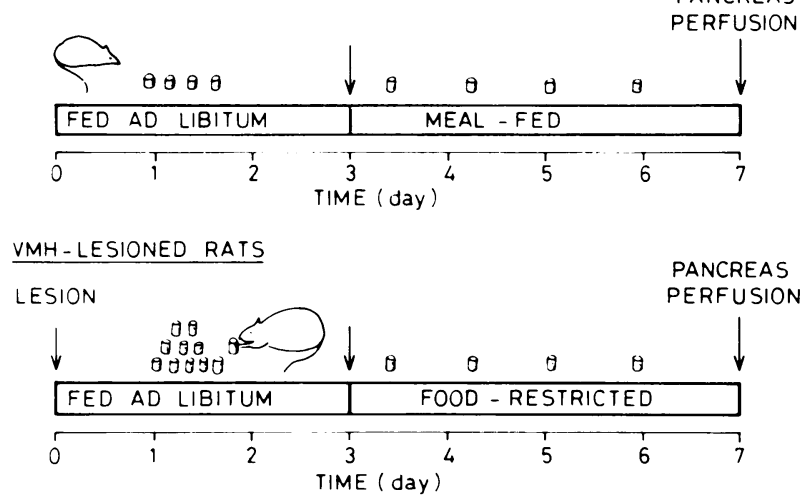

B
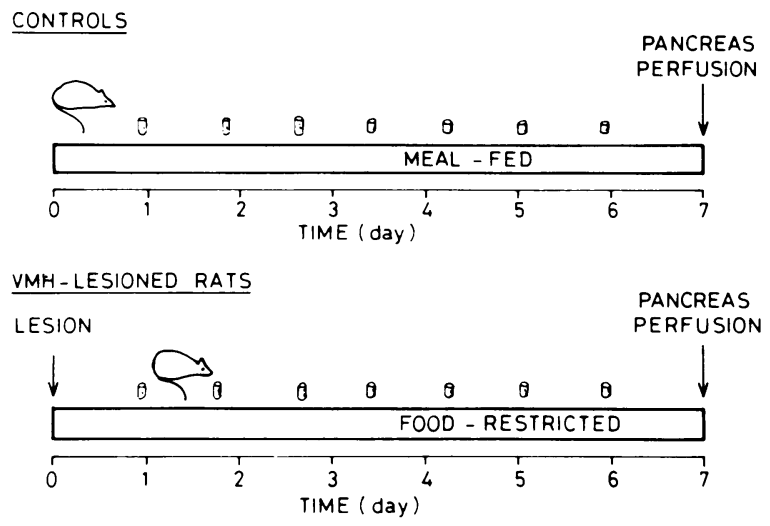

FIGURE 1 Experimental designs used for preventing hyperphagia of VMH-lesioned rats before pancreas perfusion. (A) Prevention of hyperphagia after $3 \mathrm{~d}$. (B) Immediate prevention of hyperphagia. See Methods for details.

food intake of normal rats at this age. This made the avidity for food at distribution time similar (although not identical) in control and V.MH-lesioned rats. At the end of this 7 -d period, the body weights of VMH-lesioned animals were significantly greater than those of controls as was total carcass lipid content (Table I); moreover, basal insulinemia measured 4 $\mathrm{d}$ after lesioning was greater in $\mathrm{VMH}$-lesioned rats than in controls. VMH-lesioned animals that did not increase their body weight by at least $30 \mathrm{~g}$ during the first $3 \mathrm{~d}$ of hyperphagia (control rats increased their body weight by 6-9 g during the same period), and which had basal plasma insulin level $<6 \mathrm{ng} / \mathrm{ml}$, were discarded.

C. In the third experimental condition, $8 \mathrm{wk}$-old rats were also used. To avoid the initial phase of hyperphagia, VMHlesioned animals were immediately placed in the automatic food distributor for a 7-d period (Fig. 1B). At the end of this period, and as shown by Table I, the body weights of VMH-lesioned animals were identical to those of controls, although total carcass lipid content was significantly increased in V.MH-lesioned rats when compared with controls. Because diagnosis of successful lesions of the VMH area could no 
TABLE I

Basic Characteristics of VMH-lesioned Rats

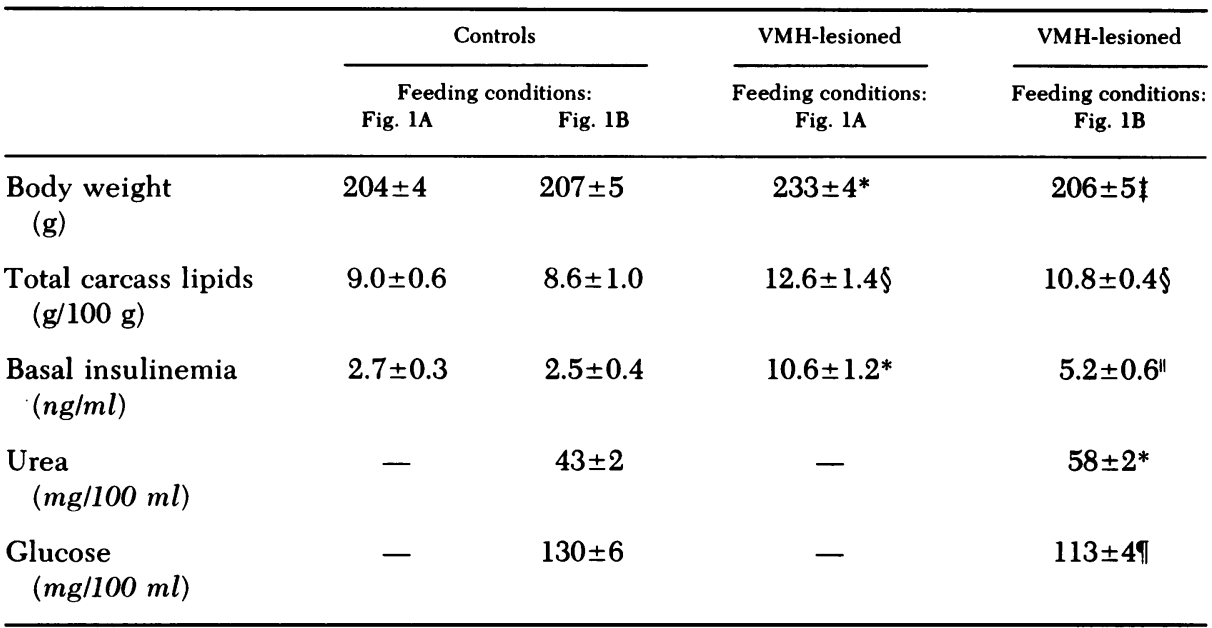

Basal insulinemia, plasma urea, and glucose levels were measured on the 4th $\mathrm{d}$ after VMH lesions, $4 \mathrm{~h}$ after the last meal given by the automatic food distributor. Each figure is the mean of $12-16$ values \pm SEM.

Statistical analysis: controls vs. VMH-lesioned:

${ }^{*} P<0.0005 ; \ddagger=\mathrm{NS} ; \S P<0.025 ; " P<0.005 ; \uparrow P<0.01$.

longer be made on the basis of initial hyperphagia and increased body weight, three other criteria were used. On the 4th $\mathrm{d}$ after the VMH lesions, i.e., $3 \mathrm{~d}$ before pancreas perfusion, blood was taken from a tail vein for plasma insulin, urea, and glucose measurements. As seen in Table I, adequate lesions of the VMH could be determined, in such animals that were never hyperphagic, by the observations of consistent increases in both plasma insulin and urea levels (the latter observation has been previously reported $[26,29,30])$, and decrease in glycemia. The first two criteria were most reliable and were used to decide which VMHlesioned rats could be used for pancreas perfusion. VMHlesioned rats with basal insulinemia $<3.5 \mathrm{ng} / \mathrm{ml}$ and plasma urea levels $<49 \mathrm{mg} / 100 \mathrm{ml}$ were discarded, the rejection percentage being $\sim 40 \%$ of total lesioned rats.

For the three different experimental conditions $\mathrm{A}$ to $\mathrm{C}$, the percentage of rejection of $\mathrm{VMH}$-lesioned rats was similar, i.e., $40 \%$. The subsequent histological examination of brains, carried out in all animals, revealed that in the rejected ones, the VMH lesions were usually not complete and asymmetrical with respect to the third ventricle. In rare instances, $(\sim 2 \%$ of the total rejected rats) rejected VMH-lesioned rats had adequate and complete VMH lesions. This is in keeping with the recent observation that successful and unsuccessful VMH lesions (in terms of their capacity to produce hyperinsulinemia) are not necessarily different in size and location but could depend upon damaging critical fibers that may or may not pass directly through the ventromedial nucleus (20).

Pancreas perfusion and perfusion medium. Rats were anesthetized with thiopentone sodium ( $80 \mathrm{mg} / \mathrm{kg}$ body $\mathrm{wt}$ ), and the pancreas was isolated and perfused according to the method of Grodsky (31) as modified by Assan et al. (32) using a non-recirculating medium gassed with $\mathrm{O}_{2} / \mathrm{CO}_{2}$ (95:5). The perfusion medium was a Krebs-Ringer bicarbonate buffer containing $0.4 \%$ bovine serum albumin (Sigma Chemical Co., St. Louis, Mo.). When glucagon secretion was measured, the perfusion medium contained $0.25 \%$ human serum albumin (Red Cross, Berne, Switzerland), and Iniprol (2,000 anti- protease U/ml, Laboratoire Choay, Paris, France). The flow rate was measured regularly and maintained at $3 \mathrm{ml} / \mathrm{min}$. Unlabeled secretagogues dissolved in perfusion medium were infused into the circuit at a rate of $0.5 \mathrm{ml} / \mathrm{min}$ (Braun infusion pump, B. Braun Apparatebau, Melsungen, Germany) $15 \mathrm{~cm}$ away from the pancreas to allow adequate mixing with the perfusion medium. Total flow was therefore $3.5 \mathrm{ml} / \mathrm{min}$. In all experiments, pancreases were perfused for $35 \mathrm{~min}$ before addition of the secretagogue, with a stimulation period lasting for $20 \mathrm{~min}$. For the kinetic studies of hormone release, perfusion samples were collected at various time intervals from the cannula leaving the portal vein, cooled on ice, and stored at $-20^{\circ} \mathrm{C}$. The secretagogues used for insulin or glucagon secretion were either glucose $(20 \mathrm{mM})$ or arginine $(10 \mathrm{mM})$. The rate of the hormone release was calculated by multiplying the hormonal concentration of the respective samples by the flow rate. In some cases, when patterns of hormonal secretion were not evaluated, the whole effluent was collected during a 20 -min stimulation period to measure total hormone output. In these experiments the secretatogues were glucose (3-19 $\mathrm{mM}$ ) for insulin release, and epinephrine $(0.1 \mu \mathrm{M})$ for that of glucagon. The functional integrity of the pancreases was assessed by: $(a)$ the constancy of perfusion pressure over the whole experiment time $(\sim 80$ $\mathrm{mm} \mathrm{Hg}) ;(b)$ adequate $\mathrm{O}_{2}$ consumption $(80-140 \mathrm{~mm} \mathrm{Hg}) ;(c)$ presence of duodenal peristaltic activity; $(d)$ presence (not shown in the figures) of a rebound hormonal secretion following a bolus of secretagogue at the end of the stimulation period; $(e)$ even distribution of trypan blue dye infused into the preparation at the end of the experiment. Pancreases that did not meet these five criteria were discarded and the rejection percentage was about $10 \%$. Results have been expressed per total pancreases, as pancreases from both groups had identical wet weights (controls: $0.87 \pm 0.03 \mathrm{~g}$; VMHlesioned: $0.83 \pm 0.03, n=14$, NS). The Student $t$ test for unpaired data was used throughout the study for comparison of mean values.

Immunoassays. Aliquots of medium were analyzed for 
insulin according to Herbert et al. (33), using rat insulin as standard, and for glucagon according to Unger et al. (34), using the $30 \mathrm{~K}$ antibody specific for pancreatic glucagon and porcine-glucagon standards.

Biochemical measurements. Plasma glucose levels were measured by the glucose oxidase method (35) and plasma urea by the urease technique (36). The total lipid content of the carcasses was measured, after homogenization of the animals, according to the method of Folch et al. (37).

Chemicals. All organic and inorganic chemicals were of analytical grade and purchased from Merck AG (Darmstadt, Germany) or from Sigma Chemical Co.

\section{RESULTS}

Insulin secretion. As illustrated by Fig. 2, basal insulin release in the presence of $5 \mathrm{mM}$ glucose was significantly higher in pancreases from VMH-lesioned rats fed ad lib. for $4 \mathrm{~d}$ than in controls. An increase of the glucose concentration in the perfusion medium to $20 \mathrm{mM}$ markedly stimulated insulin secretion in control pancreases with the typical biphasic pattern. Insulin secretion decreased when glucose concentration was reduced from 20 to $5 \mathrm{mM}$. In pancreases from $\mathrm{VMH}-$ lesioned rats fed ad lib. for $4 \mathrm{~d}$, the pattern of insulin secretion was qualitatively similar to that of controls after the addition of $20 \mathrm{mM}$ glucose, but the total insulin output was much greater and remained at a higher level than in controls when glucose concentration in the perfusate was lowered to $5 \mathrm{mM}$.

Because the excessive insulin secretion observed in pancreases from VMH-lesioned rats fed ad lib. might be the result of hyperphagia, the latter was prevented by using the first experimental design schematized by
Fig. 1A (i.e., $4 \mathrm{~d}$ of food restriction). Under these conditions, basal insulin secretion $(5 \mathrm{mM}$ glucose) by pancreases from VMH-lesioned rats was identical to controls, as shown by Fig. 3. Increasing the glucose concentration from 5 to $20 \mathrm{mM}$ again caused greater insulin output by pancreases from $\mathrm{VMH}$-lesioned rats when compared with controls, the biphasic pattern of the secretion of the hormone being preserved. By using animals with the same feeding conditions before perfusion (Fig. 1A), we tested insulin secretion elicited by various glucose concentrations. In these experiments, the total amount of insulin released during a $20-\mathrm{min}$ perfusion period was measured. As shown in Fig. 4 total insulin output was similar in both groups at $3 \mathrm{mM}$ glucose but, from $7 \mathrm{mM}$ onward, pancreases from VMH-lesioned rats consistently released more insulin than controls.

To further substantiate the observation that increased output of insulin by pancreases from $\mathrm{VMH}$ lesioned rats was not a result of the 3-d initial hyperphagia (Fig. 1A), the experimental protocol was modified so that food restriction started immediately after the VMH lesions (Fig. 1B). Successful VMH lesions were diagnosed as described in Methods. As illustrated by Fig. 5 , insulin secretion by perfused pancreases from VMH-lesioned rats that were never hyperphagic remained qualitatively normal (i.e., biphasic) but, following a glucose challenge, was still quantitatively greater than that of controls. Table II summarizes the calculated net insulin secretion above base line of all experiments. Regardless of the feeding paradigm used, pancreases from VMH-lesioned rats always responded

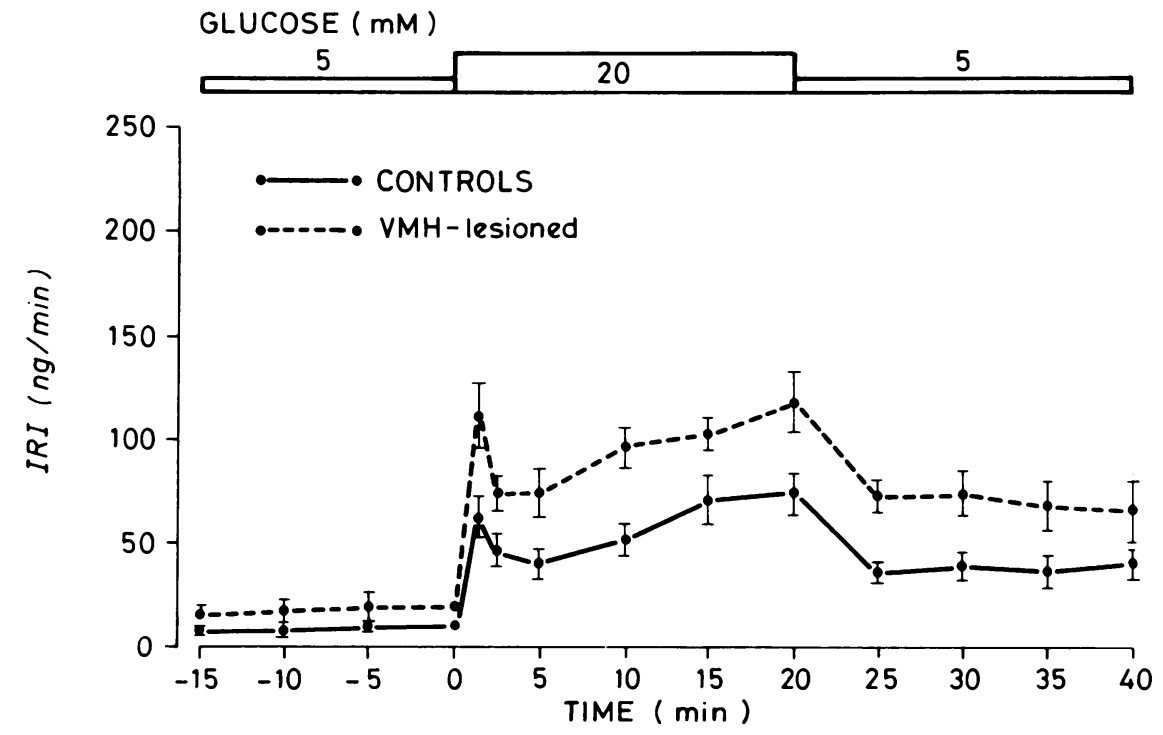

FIGURE 2 Immunoreactive insulin (IRI) release by perfused pancreases from $4 \mathrm{~d}$ ad lib.-fed control and VMH-lesioned rats. Perfusion medium was a Krebs-Ringer bicarbonate buffer with bovine serum albumin $(0.4 \%)$ and glucose as indicated. Each point, mean \pm SEM of 14 (controls) and 5 (VMH-lesioned) experiments. For statistical analysis of total IRI output, see Table II. 


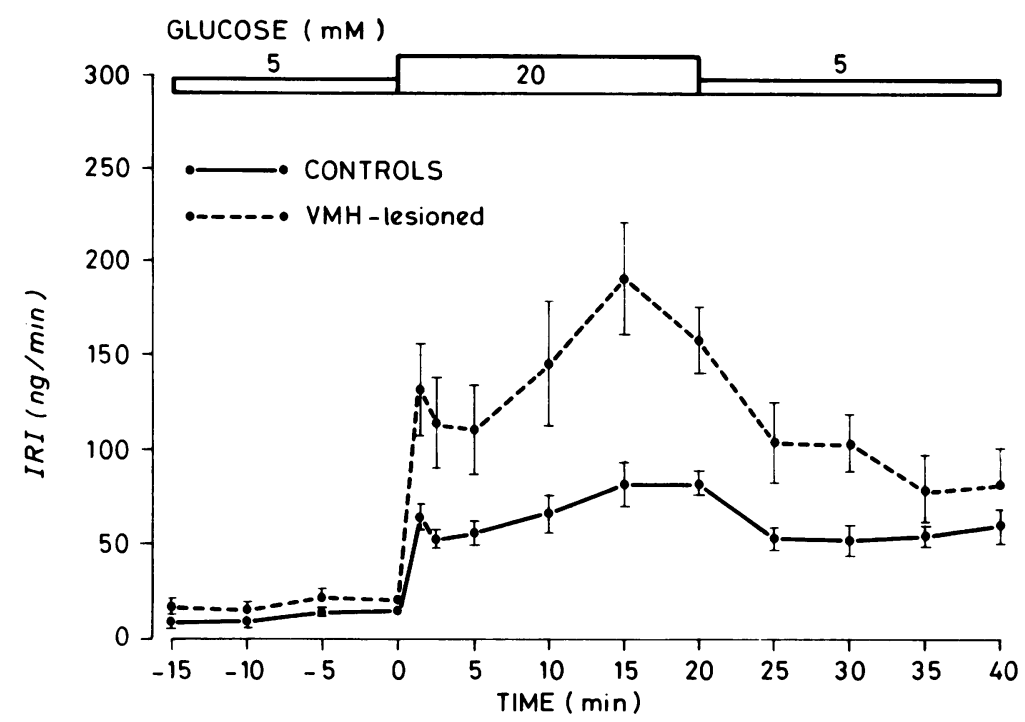

FIGURE 3 IRI release by perfused pancreases from fed controls and VMH-lesioned rats fed a restricted diet for $4 \mathrm{~d}$ matching that of controls (see experimental design, Fig. 1A). Perfusion medium as in Fig. 2. Each point, mean \pm SEM of six (controls) and seven (VMH-lesioned) experiments. For statistical analysis of total IRI output, see Table II.

to a glucose challenge by an insulin secretion that was markedly greater than that of controls.

Glucagon secretion. The first experiments were carried out using the experimental design shown by

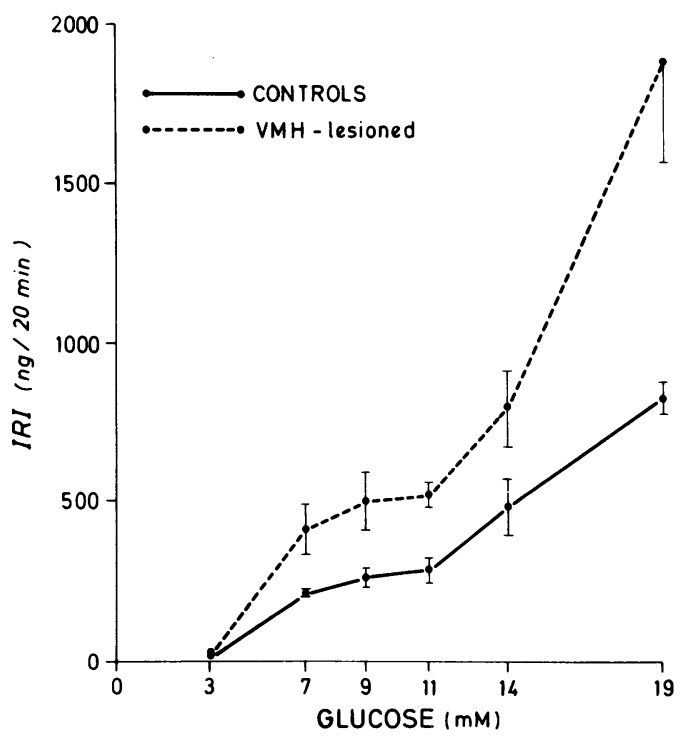

FIGURE 4 IRI release as a function of glucose concentration in the medium by perfused pancreases from fed controls and $\mathrm{VMH}$-lesioned rats fed a restricted diet for $4 \mathrm{~d}$ matching that of controls (see experimental design, Fig. 1A). Perfusion medium was as in Fig. 2. Total insulin output was measured by collecting the whole effluent during a 20 -min perfusion in the presence of glucose at the indicated concentrations. Each point, mean \pm SEM of four to five experiments. All differences were statistically significant at at least $P<0.05$ from $7 \mathrm{mM}$ onward.
Fig. 1A (i.e., 4-d food-restricted VMH-lesioned rats). As shown in Fig. 6, during the prestimulation period, basal immunoreactive glucagon (IRG) secretion by pancreases from VMH-lesioned rats was greater than that of controls. The addition of arginine $(10 \mathrm{mM})$ to control pancreases or to pancreases from VMH-lesioned rats produced the typical biphasic pattern of glucagon release. Although the first phase glucagon release was identical in both groups, the second phase was clearly

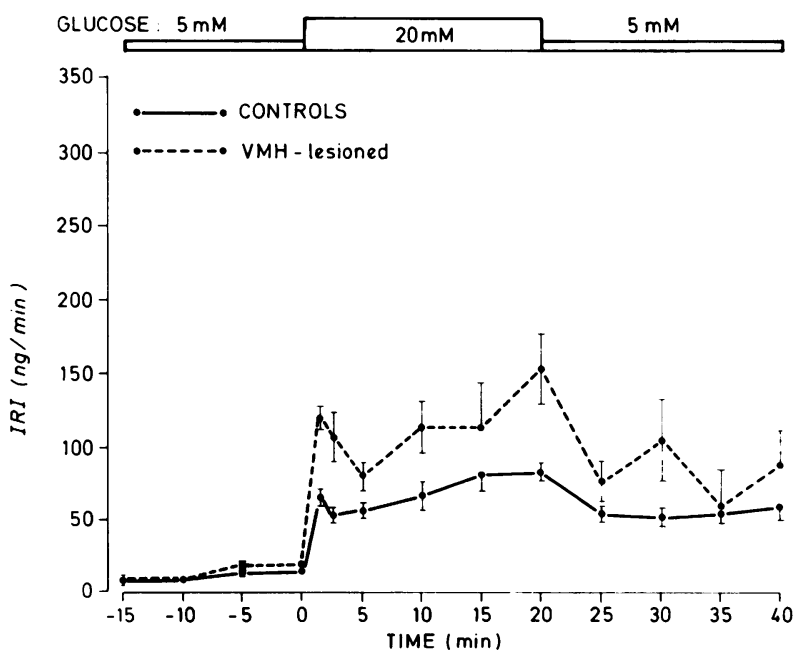

FIGURE 5 IRI release by perfused pancreases from immediately food-restricted control and VMH-lesioned rats (see experimental design, Fig. 1B). Perfusion medium was as in Fig. 2. Each point, mean \pm SEM of six (controls) and five (VMH-lesioned) experiments. For statistical analysis of total IRI output, see Table II. 


\section{TABLE II}

Calculated Net IRI Secretion Minus Base Line by Perfused Pancreases from Control and VMH-lesioned Rats Tested under Varying Feeding Conditions

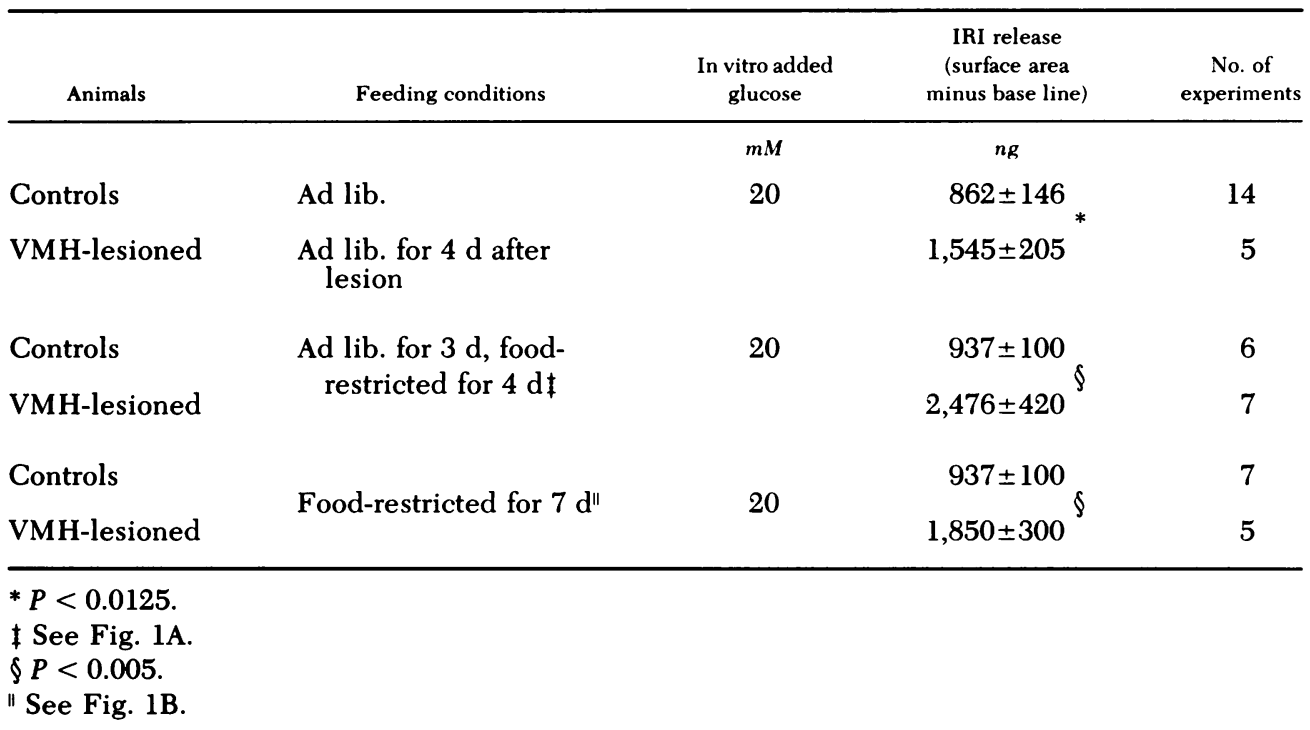

increased in pancreases from VMH-lesioned rats when compared with controls. The effect of the high arginine concentration was not the result of changes in osmolarity of the perfusion medium, as proposed elsewhere (38), because increasing osmolarity by the addition of $20 \mathrm{mM}$ saccharose completely failed to alter the secretion of glucagon by perfused pancreases of both groups (data not shown). The increased glucagon output observed in pancreases from VMH-lesioned rats fed according to Fig. 1A was not restricted to arginine stimulation and could also be observed with epinephrine, as shown by Fig. 7. In other experiments, VMHlesioned rats were immediately food-restricted (Fig. 1B) and their pancreases perfused again in the presence of epinephrine. From Fig. 7 it is clear that total glucagon secretion over a 20 -min period in the presence of epinephrine was also greater in pancreases from immediately food-restricted VMH-lesioned rats than in controls, indicating that such finding could be obtained with either one of the two feeding paradigms.

\section{DISCUSSION}

These experiments, using an in vitro system, confirm and extend experiments carried out in our own $(20,28$, $30)$ and in other laboratories $(12-15,39)$ suggesting a functional relationship between some hypothalamic area and the activity of the endocrine pancreas.

Isolated perfused pancreases obtained from $\mathrm{VMH}$ lesioned rats that were either fed ad lib. or foodrestricted to an amount of food matching that of controls either after $3 \mathrm{~d}$ of hyperphagia or immediately, always responded to a glucose challenge by an oversecretion of insulin without alteration of the biphasic pattern of the hormone release. Furthermore, total insulin secretion during a 20-min stimulation with a glucose concentration in the perfusate above $7 \mathrm{mM}$ was always higher in pancreases from $\mathrm{VMH}$-lesioned rats than in control pancreases.

Another important finding in this study was that glucagon was also oversecreted by pancreases from VMH-lesioned rats. Indeed, arginine stimulation resulted in a twofold increase in total glucagon output in perfused pancreases from VMH-lesioned rats that had been food-restricted after $3 \mathrm{~d}$ of hyperphagia when compared with their respective controls. In addition, pancreases from VMH-lesioned rats that were either food-restricted after $3 \mathrm{~d}$ of hyperphagia or immediately food-restricted (Fig. 1A, 1B) overresponded identically to an epinephrine stimulation. This indicated that both experimental protocols were valid and that the initial hyperphagia was of little subsequent consequence.

Reports on peripheral plasma glucagon levels in VMH-lesioned rats are scant and contradictory because either a decrease (40) or an increase (30) in basal levels has been reported. Furthermore, little data are available on the hepatic clearance of glucagon in normal rats (41) and none in VMH-lesioned animals. Our perfusion data are compatible with what may happen in the portal vein in vivo. Indeed, preliminary observations of an increase in portal levels of both insulin and glucagon in anesthetized VMH-lesioned rats when compared with controls have been reported. ${ }^{2}$

\footnotetext{
${ }^{2}$ Bobbioni, E., and C. Coscelli. Insulin and glucagon response to arginine in portal blood of VMH rats. Horm. and Metab. Res. In press.
} 


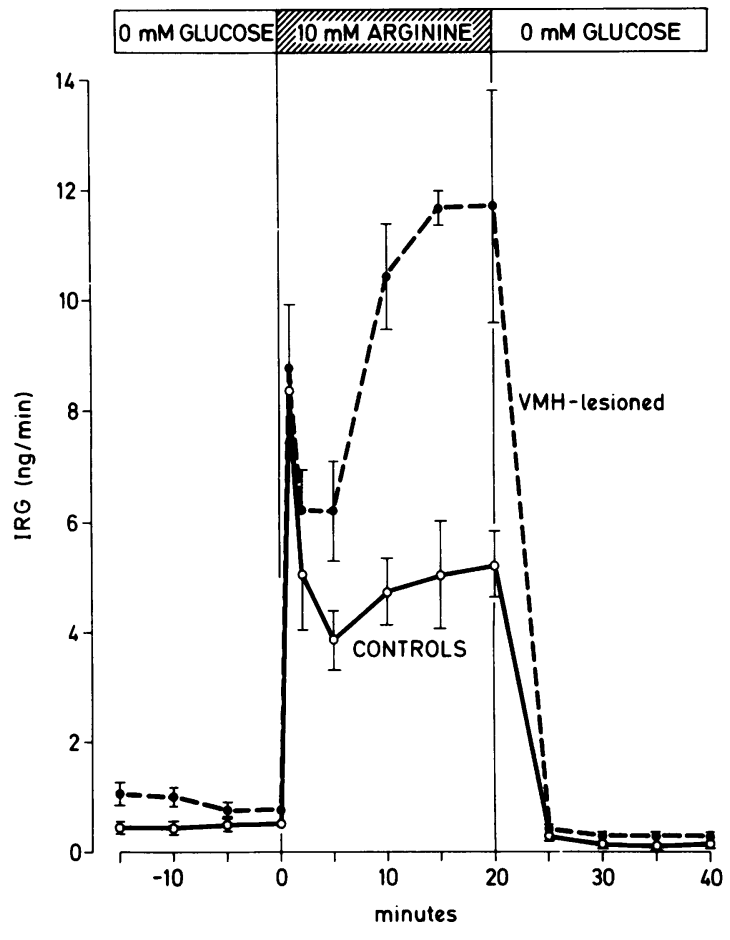

FIGURE 6 IRG release by perfused pancreases from fed controls and VMH-lesioned rats fed a restricted diet for $4 \mathrm{~d}$ matching that of controls (see experimental design, Fig. 1A). Perfusion medium was a Krebs-Ringer bicarbonate buffer with human serum albumin $(0.25 \%)$ plus Iniprol (2,000 antiprotease $\mathrm{U} / \mathrm{ml}$ ), and arginine as indicated. Basal IRG secretion (0 glucose) was significantly different between the two groups $(P<0.025)$. First IRG peak: control vs. VMH-lesioned: NS. Calculated net IRG secretion above base line was $86 \pm 12$ (controls) and $172 \pm 5 \mathrm{ng}$ (VMH-lesioned), significant at $P<0.0005$. Each point, mean \pm SEM of six (controls) and eight (VMH-lesioned) experiments.

It has also been suggested that livers from $\mathrm{VMH}-$ lesioned rats could be overstimulated by both insulin and glucagon (26).

The true nature of the changes observed in pancreases from VMH-lesioned rats is still ill-defined. As mentioned in the introduction, it has been strongly suggested that the vagus nerve was involved in the development of the VMH syndrome. It could therefore be postulated that a trophic action of the vagus nerve on the endocrine pancreas would prevail in VMHlesioned rats that would result, with time, in an increase of the size and/or number of the islets. This is in keeping with the observation of increased islet size in food-restricted VMH-lesioned rats (42). Such increased islet size (or number) could, in particular, be responsible for the changes observed in insulin and glucagon secretion. It is also possible that in VMH-lesioned rats the various endocrine cells of the pancreas would be altered in their respective putative interactions. Indeed, it has been shown recently that, when compared

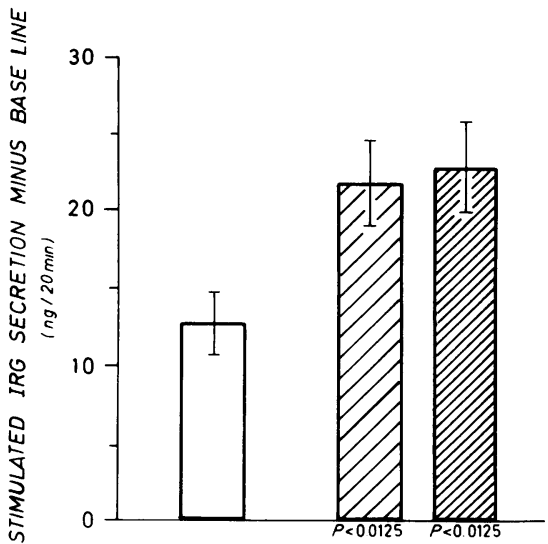

FIGURE 7 Epinephrine-stimulated IRG release by perfused pancreases from fed controls, VMH-lesioned rats, foodrestricted as indicated. Perfusion medium was as in Fig. 6. Glucagon output was measured by collecting the whole effluent during a 20 -min perfusion in the presence of epinephrine $(0.1 \mu \mathrm{M})$ plus glucose $(2.3 \mathrm{mM})$. Basal IRG secretion was $0.73 \pm 0.05 \mathrm{ng} / \mathrm{min}$ (controls), $0.83 \pm 0.12 \mathrm{ng} / \mathrm{min}$ (VMHlesioned fed as indicated in Fig. 1A), and $0.98 \pm 0.13 \mathrm{ng} / \mathrm{min}$ (VMH-lesioned fed as indicated in Fig. 1B). Intergroup basal IRG secretion: NS. Each bar, mean \pm SEM of 9 (controls), 11 (VMH-lesioned, Fig. 1A), and 8 (VMH-lesioned, Fig. 1B) experiments. $P$ values indicated: control vs. VMH-lesioned rats. $\square$, controls; $\square$, VMH-lesioned (ad lib. $3 \mathrm{~d}$, food-restricted $4 \mathrm{~d}$ ); $\mathbb{Z}, \mathrm{VMH}$-lesioned and food-restricted, $7 \mathrm{~d}$.

with controls, pancreases from VMH-lesioned rats secreted more insulin and glucagon but less somatostatin in response to an arginine stimulation. These changes were returned to normal upon addition of the cholinergic inhibitor, atropine (43). Thus it is mechanistically conceivable that the somatostatin-containing cell (Dcell) responsiveness of pancreases from $\mathrm{VMH}$-lesioned rats would modulate the release of insulin and glucagon, and that the changes observed in the secretory activity of these pancreases would be related to an increased cholinergic activity.

A final possibility would be the involvement of various humoral factors that would chronically stimulate the $\mathrm{A}$ and $\mathrm{B}$ cells from VMH-lesioned rats, an effect that would persist in isolated perfused pancreases. Increased gastrointestinal secretion could occur in VMH-lesioned rats either due to the reported increased gastric retention in the VMH syndrome (3) or via a direct effect of the vagus nerve. Some gastrointestinal hormones are known to stimulate insulin release, e.g., gastric inhibitory polypeptide (44) which, interestingly, has also been found in pancreatic A cells (45). The existence of a factor arising from the ventrolateral area of the hypothalamus which triggers insulin output (46-48) when added to isolated islets should also be mentioned. It is not known as yet whether such a factor would be released and play a role in the changes observed in pancreases from VMH-lesioned rats. 
In conclusion, the present study showed that $\mathrm{VMH}$ lesions brought about an increase of both insulin and glucagon secretion, changes that were unrelated to hyperphagia as the latter was prevented. Although the precise mechanism(s) by which VMH lesions produce these changes are, as mentioned above, still unsettled, these observations add additional evidences for the existence of a link between the hypothalamus and the function of the endocrine pancreas, implicating at least the $\mathrm{A}$ and $\mathrm{B}$ cells.

\section{ACKNOWLEDGMENTS}

We are greatly indebted to Dr. R. Assan (Service de Diabétologie, Hôtel Dieu, Paris) who taught us the pancreas perfusion technique, and to Professor E. S. Horton (Clinical Research Center, University of Vermont, Burlington, Vt.) for his valuable advice. We are also grateful to Miss Danièle Berry and Miss Christine Grillet for their excellent technical assistance, and to Mrs. Christiane McVeigh for having typed this manuscript.

This work was supported by grant 3.154.0.77 of the Swiss National Science Foundation, Berne, by a grant-in-aid of Nestlé SA, Vevey, Switzerland, and by grant 1 R01 AM25220-01 of the National Institutes of Health, Bethesda, Md.

\section{REFERENCES}

1. Brobeck, J. R. 1946. Mechanism of the development of obesity in animals with hypothalamic lesions. Physiol. Rev. 26: 541-559.

2. Marshall, N. B., R. J. Barrnett, and J. Mayer. 1955. Hypothalamic lesions in goldthioglucose injected mice. Proc. Soc. Exp. Biol. Med. 90: 240-244.

3. Bray, G. A., and T. F. Gallagher, Jr. 1975. Manifestation of hypothalamic obesity in man: a comprehensive investigation of eight patients and a review of the literature. Medicine (Baltimore). 54: 301-330.

4. Assimacopoulos-Jeannet, F., and B. Jeanrenaud. 1976. The hormonal and metabolic basis of experimental obesity. In Clinics in Endocrinology and Metabolism, M. J. Albrink, editor. W. B. Saunders Company, Philadelphia. Obesity. 5(II): $337-365$.

5. Brobeck, J. R., J. Tepperman, and C. N. H. Long. 1943. Experimental hypothalamic hyperphagia in the albino rats. Yale J. Biol. Med. 15: 831-853.

6. Han, P. W. 1967. Hypothalamic obesity in rats without hyperphagia. Trans. N. Y. Acad. Sci. Series II. 30: 229-243.

7. Goldman, J. K., and L. L. Bernardis. 1974. Metabolism of glucose, fructose and pyruvate in tissues of weanling rats with hypothalamic obesity. Horm. Metab. Res. 6: 454-456.

8. Frohman, L. A., and L. L. Bernardis. 1968. Growth hormone and insulin levels in weanling rats with ventromedial hypothalamic lesions. Endocrinology. 82: 11251132.

9. Han, P. W. 1968. Growth hormone effect on weanling rats with hypothalamic lesions. Chin. J. Physiol. 20: $101-108$.

10. Bogdanove, E. M., B. N. Spirtos, and N. S. Halmi. 1955. Further observations on pituitary structure and function in rats bearing hypothalamic lesions. Endocrinology. 57: $302-315$.

11. Hinman, D. J., and D. R. Griffith. 1973. Effects of ventro- medial hypothalamic lesions on thyroid secretion rate in rats. Horm. Metab. Res. 5: 48-50.

12. Hales, C. N., and G. C. Kennedy. 1964. Plasma glucose, nonesterified fatty acid and insulin concentrations in hypothalamic-hyperphagic rats. Biochem. J. 90: 620-624.

13. Frohman, L. A., L. L. Bernardis, J. D. Schnatz, and L. Burek. 1969. Plasma insulin and triglyceride levels after hypothalamic lesions in weanling rats. $\mathrm{Am} . J$. Physiol. 216: 1496-1501.

14. Friedman, M. I. 1972. Effects of alloxan diabetes on hypothalamic hyperphagia and obesity. Am. I. Physiol. 222: $174-178$.

15. York, D. A., and G. A. Bray. 1972. Dependence of hypothalamic obesity on insulin, the pituitary and the adrenal gland. Endocrinology. 90: 885-894.

16. Inoue, S., G. A. Bray, and Y. S. Mullen. 1977. Effect of transplantation of pancreas on development of hypothalamic obesity. Nature (Lond.). 266: 742-744.

17. Powley, T. L., and C. A. Opsahl. 1974. Ventromedial hypothalamic obesity abolished by subdiaphragmatic vagotomy. Am. J. Physiol. 226: 25-33.

18. Chikamori, K., K. Masuda, H. Izumi, K. Isaka, and U. Tezuka. 1977. Effect of vagotomy on hyperinsulinemia in obese rats with hypothalamic lesions. Endocrinol. Jpn. 24: $251-258$

19. Inoue, S., and G. A. Bray. 1977. The effects of subdiaphragmatic vagotomy in rats with ventromedial hypothalamic obesity. Endocrinology. 100: 108-114.

20. Berthoud, H-R., and B. Jeanrenaud. 1979. Acute hyperinsulinemia and its reversal by vagotomy after lesions of the ventromedial hypothalamus in anesthetized rats. Endocrinology. 105: 146-151.

21. Frohman, L. A., E. Z. Ezdinli, and R. Javid. 1967. Effect of vagotomy and vagal stimulation on insulin secretion. Diabetes. 16: 443-448.

22. Kaneto, A., K. Kosaka, and K. Nakao. 1967. Effects of stimulation of the vagus nerve on insulin secretion. Endocrinology. 80: 530-536.

23. Bloom, S. R., A. V. Edwards, and N. J. A. Vaughan. 1974. The role of the autonomic innervation in the control of glucagon release during hypoglycemia in the calf. $J$. Physiol. (Lond.). 236: 611-623.

24. Ernest, M. J., Ch.-L. Chen, and P. Feigelson. 1977. Induction of tyrosine aminotransferase synthesis in isolated liver cell suspensions. Absolute dependence of induction on glucocorticoids and glucagon or cyclic AMP. J. Biol. Chem. 252: 6783-6791.

25. Snodgrass, P. J., R. C. Lin, W. A. Müller, and T. T. Aoki. 1978. Induction of urea cycle enzymes of rat liver by glucagon. J. Biol. Chem. 253: 2748-2753.

26. Karakash, C., F. Rohner, B. E. Hustvedt, and B. Jeanrenaud. 1979. Possible etiology of reduced nitrogen retention in hypothalamic obese rats. Am. J. Physiol. In press.

27. De Groot, J. 1959. The rat forebrain in stereotaxic coordinates. Trans. R. Neth. Acad. Sci. 52: 1-40.

28. Rohner, F., A. C. Dufour, C. Karakash, Y. Le Marchand, K. B. Ruf, and B. Jeanrenaud. 1977. Immediate effect of lesion of the ventromedial hypothalamic area upon glucose-induced insulin secretion in anesthetized rats. Diabetologia. 13: 239-242.

29. Hustvedt, B. E., A. Løvø, and D. Reichl. 1976. The effect of ventromedial hypothalamic lesions on metabolism and insulin secretion in rats on a controlled feeding regimen. Nutr. Metab. 20: 264-271.

30. Karakash, C., B. E. Hustvedt, A. L $\emptyset$ $\emptyset$, Y. Le Marchand, and B. Jeanrenaud. 1977. Consequences of ventro- 
medial hypothalamic lesions on metabolism of perfused liver. Am. J. Physiol. 232: E286-E293.

31. Grodsky, G. M., L. Bennett, D. F. Smith, and F. G. Schmid. 1967. Effects of pulse administration of glucose or glucagon on insulin secretion in vitro. Metab. Clin. Exp. 16: 222-233.

32. Assan, R., J. R. Attali, G. Ballerio, J. Baillot, and J. R. Girard. 1977. Glucagon secretion induced by natural and artificial amino acids in the perfused rat pancreas. Diabetes. 26: 300-307.

33. Herbert, V., K. S. Lau, C. W. Gottlieb, and S. J. Bleicher. 1973. Coated charcoal immunoassay of insulin. J. Clin. Endocrinol. Metab. 25: 1375-1384.

34. Unger, R. H., E. Aguilar-Parada, W. A. Müller, and A. M. Eisentraut. 1970. Studies of pancreatic alpha-cell function in normal and diabetic subjects. J. Clin. Invest. 49: $837-848$.

35. Bergmeyer, H. U., and E. Bernt. 1963. In Methods of Enzymatic Analysis. H. U. Bergmeyer, Ed. Academic Press Inc., New York. 123-130.

36. Bernt, E., and H. U. Bergmeyer. 1963. In Methods of Enzymatic Analysis. H. U. Bergmeyer, Editor. Academic Press, Inc., New York. 401-406.

37. Folch, J., M. Lees, and G. H. Sloane-Stanley. 1957. A simple method for the isolation and purification of total lipids from animal tissues. J. Biol. Chem. 226: 497-509.

38. Hahn, H. J., and M. Ziegler. 1977. Investigations on isolated islets of Langerhans in vitro. 16. Modification of the glucose-dependent inhibition of glucagon secretion. Biochim. Biophys. Acta. 499: 362-372.

39. Bierwolf, B., W. Blech, and I. Weiss. 1977. Glucoseinduced insulin and glucagon secretion by the isolated perfused pancreas of Wistar rats with lesions in the ventromedial hypothalamic nuclei (VMN). Diabetologia. 13: 382. (Abstr.)

40. Bray, G. A., and S. Inoue. 1976. Hypothalamic obesity lowers glucagon. Clin. Res. 24: 426A. (Abstr.)

41. Jaspan, J. B., A. H-J. Huen, C. G. Morley, A. R. Moossa, and A. H. Rubenstein. 1977. The role of the liver in glucagon metabolism. J. Clin. Invest. 60: 421-428.

42. Han, P. W., Y-K. Yu, and S. L. Chow. 1970. Enlarged pancreatic islets of tube-fed hypophysectomized rats bearing hypothalamic lesions. Am. J. Physiol. 218: 769-771.

43. Rohner, F., and B. Jeanrenaud. 1979. Abnormalities of the endocrine pancreas following hypothalamic lesions in the rat. Experientia (Basel). 35: 942. (Abstr.)

44. Creutzfeldt, W., R. Ebert, B. Willms, H. Frerichs, and J. C. Brown. 1978. Gastric inhibitory polypeptide (GIP) and insulin in obesity: Increased response to stimulation and defective feedback control of serum levels. Diabetologia. 14: 15-24.

45. Smith, P. H., F. W. Merchant, D. G. Johnson, W. Y. Fujimoto, and R. H. Williams. 1977. Immunocytochemical localization of a gastric inhibitory polypeptide-like material within A-cells of the endocrine pancreas. Am. J. Anat. 149: 585-590.

46. Idahl, L. §., and J. M. Martin. 1971. Stimulation of insulin release by a ventrolateral hypothalamic factor. J. Endocrinol. 51: 601-602.

47. Hill, D. E., S. Mayes, D. Di Battista, R. Lockhart-Ewart, and J. M. Martin. 1977. Hypothalamic regulation of insulin release in rhesus monkeys. Diabetes. 26: 726-731.

48. Lockhart-Ewart, R. B., C. Mok, and J. M. Martin. 1976. Neuroendocrine control of insulin secretion. Diabetes. 25: $96-100$. 